ESJ Humanities

\title{
Dynamiques spatiales et mobilités à Akonolinga (Cameroun)
}

\author{
Mediebou Chindji \\ Chargée de cours au département de Géographie Université de Yaoundé I \\ Otsomotsi Mbida Alain Martin \\ Démographe, Ministère de l'Économie, de la Planification et de \\ l'Aménagement du Territoire, Cameroun
}

Doi:10.19044/esj.2021.v17n28p81

Submitted: 29 December 2020

Accepted: 06 July 2021

Published: 31 August 2021
Copyright 2021 Author(s)

Under Creative Commons BY-NC-ND

4.0 OPEN ACCESS

Cite As:

Mediebou C. \& Otsomotsi Mbida A. M. (2021). Dynamiques spatiales et mobilités à Akonolinga (Cameroun). European Scientific Journal, ESJ, 17 (28), 81.

https://doi.org/10.19044/esj.2021.v17n28p81

\section{Résumé}

La ville d'Akonolinga, comme presque toutes les villes camerounaises, est le théâtre des dynamiques spatiales ces trente dernières années. Les conditions et le cadre de vie de cette petite ville ainsi que les activités économiques se sont considérablement dégradées. Ce constat semble être relayé par les déclarations des personnes ayant vécues à Akonolinga dans les années 1990 qui se désolent de la situation peu reluisante de cette ville jadis prospère et vivante. La présence des migrants constitue une donnée fondamentale dans l'analyse de cette situation. Ceuxci proviennent non seulement des campagnes environnantes, mais aussi de la quasi-totalité des régions administratives du Cameroun. Ces arrivées croissantes et continues ont des conséquences socio-économiques et environnementales. L'objectif de ce travail est de mettre en évidence l'impact de l'immigration sur la dynamique spatiale et l'accroissement de la pauvreté à Akonolinga. La méthodologie est centrée autour des enquêtes socio-économiques auprès de 342 ménages ; des interviews, des observations de terrain et la consultation de la littérature liée au thème en question. Les résultats montrent que les quartiers périphériques ont bénéficié de l'apport démographique des immigrants installés dans les quartiers centraux et péricentraux à leur arrivée. Ces migrants investissent dans les activités économiques des services et participent de fait à la création des richesses à Akonolinga. De nombreuses mesures ont été mises en œuvre pour 
limiter les déplacements des populations des zones rurales vers les zones urbaines. Ces mesures ont eu des résultats mitigés justifiant ainsi leur évaluation en vue de définir des politiques et programmes plus adaptés et à même de promouvoir un développement socioéconomique équilibré entre les villes et les campagnes.

Mots clés : Dynamiques spatiales, mobilités, immigration et pauvreté

\title{
Spatial dynamics and mobilities in Akonolinga (Cameroon)
}

\author{
Mediebou Chindji \\ Chargée de cours au département de Géographie1Université de Yaoundé I \\ Otsomotsi Mbida Alain Martin \\ Démographe, Ministère de l'Économie, de la Planification et de \\ l'Aménagement du Territoire, Cameroun
}

\begin{abstract}
During the last thirty years, socio-spatial dynamics have been observed in the urban space of Akonolinga. The conditions and living environment of this small town and its economic activities have deteriorated considerably. This observation seems to be relayed by the statements of people who lived in Akonolinga in the 1990s who are sorry for the poor condition of this once prosperous and lively city. Indeed, despite this situation, this city continued to welcome migrants. These come not only from the surrounding countryside, but also from almost all the administrative regions of Cameroon. These growing and continuous arrivals have multifarious consequences. The purpose of this work is to highlight the impact of immigration on spatial dynamics and increasing poverty in Akonolinga. The hypothetico-deductive method was mobilized and survey and census data were used for spatial and statistical analyzes. The results show that the outlying districts benefited from the demographic contribution of the immigrants settled in the central and pericentral districts on their arrival. Many measures have been implemented to limit the movement of people from rural areas to urban areas. These measures have had mixed results, justifying their evaluation with a view to defining more appropriate policies and programs that can promote balanced socioeconomic development between cities and the countryside.
\end{abstract}

Keywords: Spatial Dynamics, Mobility, Immigration and Poverty 


\section{Introduction}

Durant les deux dernières décennies, la question des migrations a incontestablement pris une place croissante dans les agendas politiques d'une majorité d'états sur la planète. Les médias s'en font largement écho et les sociétés civiles ne manquent pas elles aussi de réagir. La migration est un phénomène humain complexe. Pour en comprendre les causes, il est indispensable de l'étudier en tenant compte du niveau de développement des sociétés de départ et de destination. Même si les relations entre migration et développement sont complexes, dans le sens où la migration n'est pas simplement la conséquence directe de la pauvreté, elle est toujours corrélée à l'état de développement des sociétés (Oliver Bakewell et al, 2011) . Par conséquent, il est difficile de comprendre la migration sans envisager les dynamiques relatives aux changements socio-spatiaux qui affectent à la fois les zones de départ et celles de destination des migrants comme le soulignent dans leurs travaux Jacquet (2012) et la Conférence des Nations Unies sur le Commerce et le Développement (2018).

Dans leur trajectoire pour une quête du mieux-être, les migrants internes des pays du Sud comme le Cameroun préfèrent toujours les zones urbaines au détriment des espaces ruraux de plus en plus pauvres (Teye et al, 2020). A titre illustratif, 53,6\% de la population de Douala et $51.9 \%$ de celle de Yaoundé sont des migrants. Par ailleurs, les Régions du Littoral, du Sud et du Nord sont également des pôles importants d'attraction. A l'opposé, celles de l'Extrême-Nord et de l'Adamaoua présentent un faible pouvoir attractif pour les migrants (INS, 2005). Ces déplacements vers les pôles urbains importants sont en fait l'aboutissement d'un parcours conduisant le migrant de son village d'origine vers les localités rurales présentant des opportunités (emplois, éducation, santé...), puis vers la petite ville la plus proche, la capitale régionale et enfin la grande ville (Abêt Mongbet, 2019). La ville d'Akonolinga qui est plutôt une ville de taille moyenne au Cameroun reçoit les migrants venants de toutes les régions du pays. Ses potentialités économiques sont plutôt limitées par rapport à celles de Douala, Yaoundé ou de Bafoussam.

La population d'Akonolinga est passée de 6800 habitants à 25669, soit une augmentation de $13,50 \%$ en valeur relative au cours de cette période. Cette croissance urbaine trouverait l'une de ses origines dans les mouvements migratoires internes dirigés des campagnes vers les villes (OIM, 2009). La permanence des flux migratoires en direction des villes a un impact sur leur extension spatiale et sur les phénomènes sociaux. En effet, l'urbanisation, considérée comme une conséquence de la migration aurait une influence sur les caractéristiques sociodémographiques des habitants des villes (Tabutin, 2009). L'empreinte spatiale de la migration interne à Akonolinga est perceptible sur les dynamiques socio-économiques de cette 
ville et l'impact spatial des migrants est plus analysé à travers notamment l'étalement urbain et la conquête de nouveaux espaces comme le souligne Véron (2008). Les impacts de cet afflux de population sur l'environnement urbain, notamment à travers les questions de traitement des déchets, de pollution de l'eau, de gestion sanitaire ou encore d'occupation des terres incultes périphériques sont aussi préoccupant à Akonoliga comme dans d'autres villes qui accueillent les migrants (Sandron et al, 2015). Ces migrants sont aussi des acteurs positifs es dynamiques spatiales quand ils s'illustrent dans la participation citoyenne au développement socioéconomique de la ville d'Akonolinga en contribuant à la création des richesses de toutes sortes. La dynamique spatiale et socio-économique des espaces périphériques de la ville d'Akonolinga est tenue en grande partie par les migrants car les populations autochtones sont beaucoup concentrées au centre-ville. Donc la migration est l'un des principaux facteurs de l'extension urbaine d'Akonolinga.

L'étude portant sur la relation dynamique spatiales et mobilités dans la ville d'Akonolinga vise à apporter une contribution à l'analyse globale des impacts de la migration sur l'urbanisation au Cameroun. Elle entend spécifiquement mettre à jour la prise en compte des effets des déplacements des populations sur l'espace territorial de la ville d'Akonolinga, dans un contexte où, les petites villes sont encore faiblement représentées dans les études des migrations internes. Elle repose sur l'hypothèse selon laquelle les migrants contribuent à la dynamique et la mobilité spatiales dans la ville d'Akonolinga. 


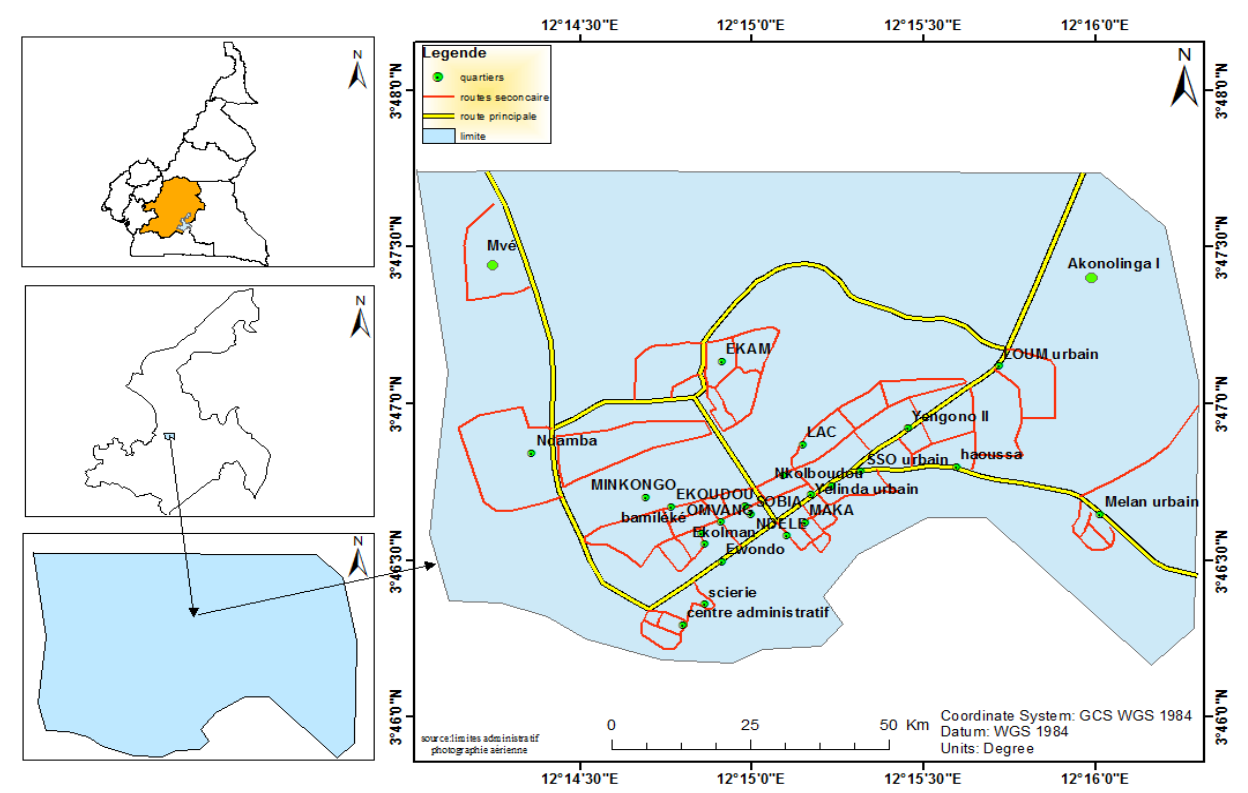

Figure 1: Localisation de la ville d'Akonolinga

\section{Méthodologie}

Pour réaliser cette étude, la méthode hypothético-déductive a été mobilisée et les données d'enquêtes socio-économiques de la population cible et des données issues du RGPH dont les résultats ont été rendus publics en 2010

L'analyse spatiale des interactions migration-dynamique spatialepauvreté a reposé sur une approche méthodologique comprenant la collecte des données, l'échantillonnage et le traitement des données et les difficultés rencontrées. Les images satellites, les photographies aériennes, les cartes thématiques ainsi que les données sur la ville d'Akonolinga ont été obtenues auprès des Archives Nationales, des archives de la Commune d'Akonolinga, de l'Institut National de Cartographie et des sites internet comme (revue.org, Google scholar, Google Earth et Glovis).

En outre, les données sociodémographiques issues des trois derniers recensements généraux de la population et de l'habitat du Cameroun (1976, 1987 et 2005) ont été mobilisées. Ces données ont été obtenues auprès du site du projet ipums-usa de l'Université du Minnesota qui permet aux utilisateurs d'avoir accès à $10 \%$ de la base de données de toutes les enquêtes et recensements réalisés à travers le monde.

La base du calcul de la taille de notre échantillon est la répartition de la population de la ville d'Akonolinga par quartier issue du plan communal de développement actualisé en 2014. Par ailleurs, les résultats du troisième recensement du Cameroun de 2005 notamment la taille moyenne du ménage ( 5 personnes) et la population de la ville d'Akonolinga (15 069 habitants) 
ont été utilisés pour déterminer les paramètres qui ont été intégrés dans ce calcul.

L'application de cette formule donne une taille d'échantillon égale à 336 ménages à enquêter. Afin de tenir compte des éventuels cas de nonréponses, la taille de l'échantillon a été réévaluée de $2 \%$. Donnant ainsi une taille définitive de l'échantillon de 342 ménages. Cet échantillon a été ventilé dans les différents quartiers proportionnellement à leur population.

Dans le souci d'avoir une vue holistique du phénomène étudié, les données collectées par questionnaire ont été complétées par des entretiens et interviews avec les personnes ressources.

Le traitement des images et photographies aériennes s'est effectué à l'aide de logiciels de traitement d'images tels que Erdas imagine 1.1, Surfer 9, ArcGIS 10 et le package office 2007 pour les cartes. Dans le cadre de cette étude, deux groupes de variables ont été utilisés : la variable dépendante et les variables indépendantes. Le traitement des données statistiques repose préalablement sur l'évaluation de leur qualité. Elles ont été analysées grâce à l'utilisation des logiciels d'analyses statistiques tels que Word et Excel. Spad version 5.5. a permis de réaliser les Analyses Factorielles en Composantes multivariés (AFCM) et SPSS version 20.0 a servi aux tris à plat et aux analyses bivariées. Les principaux résultats montrent que l'utilisation de la classification hiérarchique serait plus pertinente.

\section{Résultats}

\section{Evolution démographique et profil des migrants à Akonolinga}

La population de la ville d'Akonolinga a connu une évolution rapide passant d'environ 3900 individus en 1960 à 19282 habitants en 2005et environ 25669 habitant en 2017 selon les estimation du BUCREP (2017) .La figure 2 illustre l'évolution démographique d'Akonolinga entre 1960 et 2005 (figure 2).

Figure 2: Evolution de la population de la ville d'Akonolinga

\section{Population}

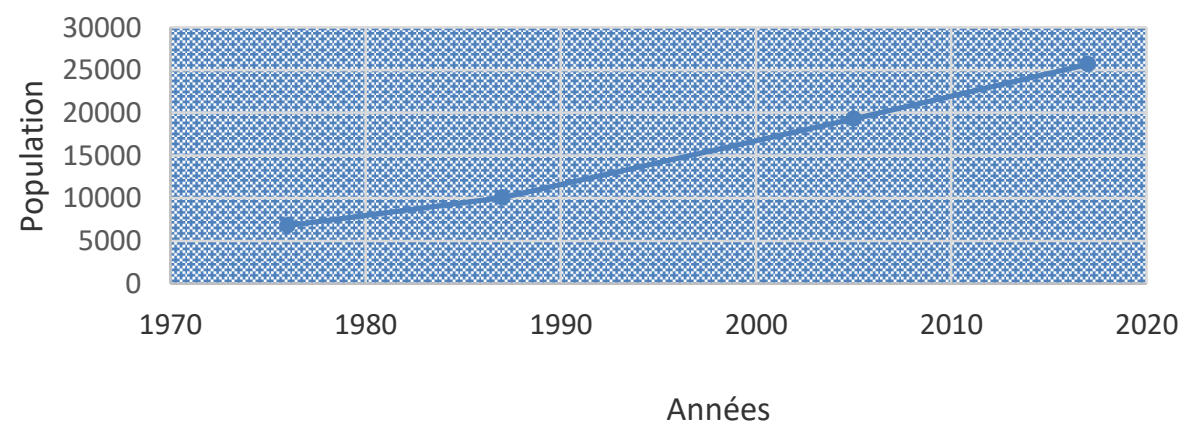

Sources : RGPH 1976, 1987 et 2005 et 2017 (projection) 
L'analyse des effectifs de cette population selon le statut migratoire montre qu'elle est essentiellement composée de migrants (tableau 1). En effet, entre 1976 et 2005, le nombre de migrants vivant à Akonolinga a été multiplié par 2,3 passant ainsi de 5773 à 13420 personnes. Cependant, on note une légère baisse de la population des migrants entre 2005 et 2017. En effet cette population est passée de 13420 à 11525 personnes (tableau 1). Cette situation est le fait d'un ralentissement des activités économiques dans la ville et de la volonté des migrants d'opter plutôt pour d'autres espaces qui offrent plus d'opportunités comme les villes frontalières du sud du pays et les régions aurifères de l'est-Cameroun.

Tableau 1 : Population de la ville d'Akonolinga selon le statut migratoire et par année

\begin{tabular}{|l|l|l|l|l|}
\hline \multirow{2}{*}{ Statut migratoire } & Année & $\mathbf{1 9 8 7}$ & $\mathbf{2 0 0 5}$ & $\mathbf{2 0 1 7}$ \\
\cline { 2 - 5 } & $\mathbf{1 9 7 6}$ & 2940 & 5862 & 14144 \\
\hline Non-migrant & 1027 & 7129 & 13420 & 11525 \\
\hline Migrant & 5773 & 10069 & 19282 & 25669 \\
\hline Total & 6800 & &
\end{tabular}

Sources : RGPH 1976, 1987 et 2005 et nos estimations sur la base des projections du BUCREP 2017

Ces migrants qui viennent de toutes les régions du Cameroun, présentent des profils qui ont évolué avec le temps et sont résumés dans les plans factoriels (annexes 3 à 6). Ainsi, en 2017 les migrants Akonolinga sont des individus dont le revenu varie en fonction des années et aussi en fonction des activités économiques menées. Ces revenus varient peu et la moyenne est de à 65000 FCFA. Ils viennent en majorité du Nyong et Mfoumou et du Mfoundi et travaillent dans le secteur primaire et secondaire. Ce sont par ailleurs des célibataires dont l'âge varie entre 15 et 29 ans.

En 2005, les migrants à Akonolinga sont en union libre, mariés, séparés/veufs ou divorcés. Ils viennent en majorité des Départements du Nyong et Mfoumou et du Dja et Lobo, sont âgés de plus de 40 ans, travaillent dans les secteurs secondaire et primaire en qualité d'employeurs ou de travailleurs indépendants ou d'employés /apprentis.

En 1987 les migrants à Akonolinga sont des hommes célibataires qui résidaient avant leur arrivée à Akonolinga dans les Départements du Nyong et Mfoumou, du Wouri et du Mfoundi. Ils travaillent dans le secteur primaire comme employés ou apprentis et ont un niveau d'instruction primaire ou secondaire.

En 1976, les migrants sont des femmes célibataires, séparées, divorcées ou veuves. Avant leur arrivée à Akonolinga, elles vivaient dans les Départements du Mfoundi, du Moungo, du Koupé-Manengounba, de la Haute-Sanaga, de la Mefou et Afamba, du Nyong et So'o, de la Vina, de la Lékié, de la Mvila, du Dja et Lobo, du Nyong et Mfoumou, de la Vallée du Ntem et du Lom et Djérem. Exerçant leurs activités dans le secteur primaire, 
elles sont âgées de 15-29 ans ou de plus de 40 ans et n'ont aucun niveau d'instruction.

La présence quasi- constante et importante des migrants dans cette ville peut être associée au fait qu'en dépit de la dégradation des conditions économiques, elle a gardé un certain attrait lié à sa fonction administrative et à l'existence d'équipements sociaux (écoles, hôpitaux...). Les migrants contribuent aussi à maintenir l'équilibre socio-économique de la ville malgré la conjoncture plutôt morose à l'épanouissement des activités économiques. Afin de mettre en exergue la relation entre la dynamique de l'espace urbanisé et la migration, nous avons établi la carte des flux migratoires en croisant les données relatives au quartier d'installation à l'arrivée à Akonolinga et celle du quartier d'installation en 2017 (figure 3).

Figure 3 : Flux migratoires dans la ville d'Akonolinga

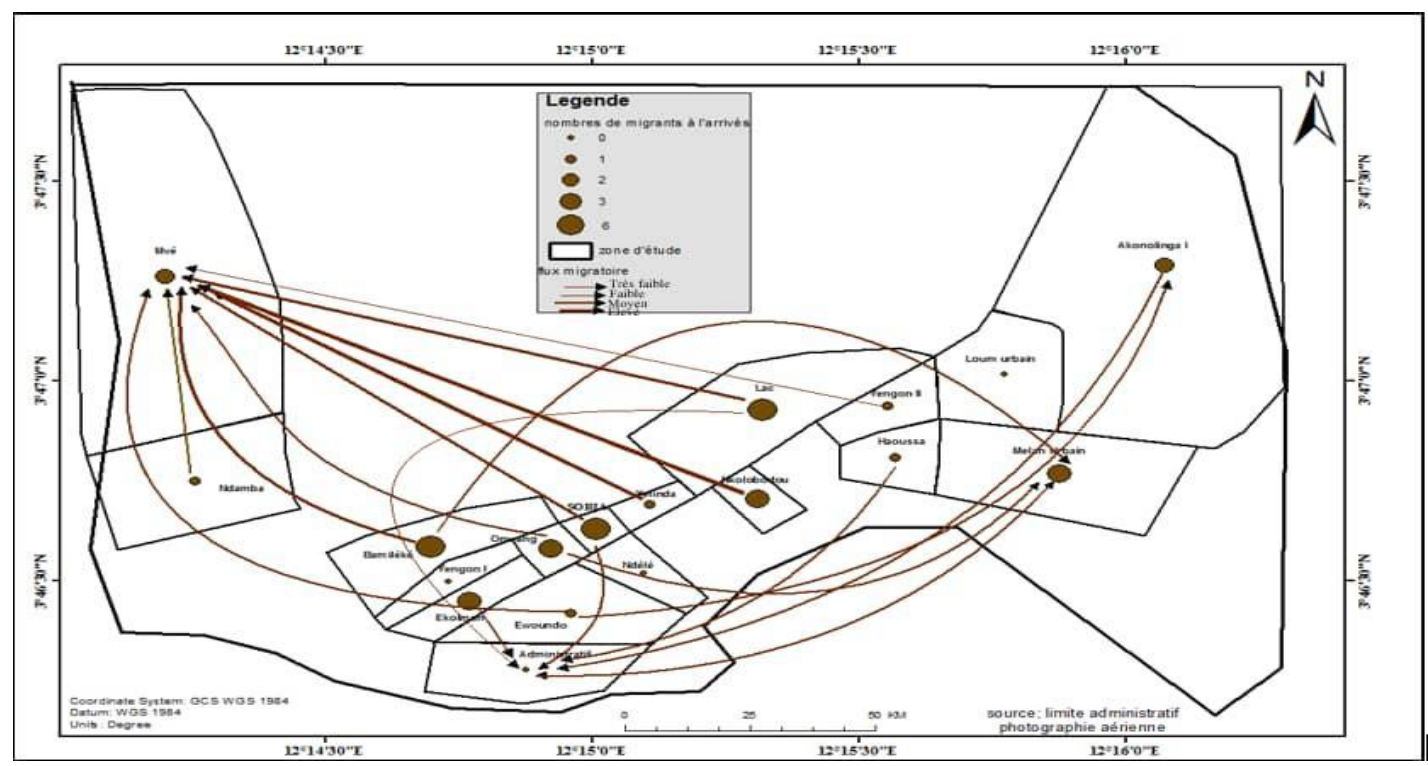

Sources : Exploitation des données de l'enquête réalisée en 2017

Les résultats présentés sur la figure 3 révèlent l'importance de la contribution des migrants à la dynamique de l'espace urbain et principalement vers le Nord-Nord-Est conséquence de leur transfert des quartiers centraux et anciens (Bamiléké, Yelinda, Lac, Sobia, Nkoloboudou) et périphériques (Ndamba, Sso) vers le quartier Mvé. Par ailleurs, nous observons aussi que le Quartier Sso bénéficie de l'arrivée des migrants installés préalablement dans d'autres quartiers (Akonolinga II, Bamiléké, Administratif). En outre, le quartier administratif bénéficie exclusivement du déplacement des migrants installés dans les quartiers anciens (Ekolman, Sobia, Haoussa). Enfin, le quartier Akonolinga ne bénéficie que des déplacements venant du quartier administratif. 


\section{Migration et dynamiques spatiales à Akonolinga}

Entre 1954 et 2017, la ville d'Akonolinga, à l'instar de tous les centres urbains du Cameroun, a connu une dynamique spatiale remarquable. Elle a ainsi été marquée par deux grandes évolutions au cours de cette période avec pour point de départ le noyau urbain de 1956. Il s'agit de la création de la « ville nouvelle » et de la densification de l'habitat.

En 1954, Akonolinga présente l'aspect d'une agglomération de forme «linéaire » caractérisée par des habitations construites de part et d'autre de l'axe routier central qui part de l'actuel carrefour du rond-point de la poste jusqu'au carrefour « Kanga » où il se sépare en deux bras à la forme d'un « Y ». Le bras de droite dessert les quartiers Haoussa et Sso tandis que celui de gauche dessert le quartier Akonolinga II. Entre le rond-point de la poste et le carrefour Kanga se font face les quartiers Ewondo, Ndele, Nkolobodou, Yengono I, Ekolman, Omvang et Yelinda. En plus de ces quartiers, on note la présence du stationnement, des commerces ainsi que la scierie.

Ce noyau originel couvrait une superficie estimée à environ 43 ha représente ainsi la ville d'Akonolinga au moment de son érection en cheflieu d'arrondissement en 1956. Il ne représentait que 6\% de l'ensemble de la superficie de la ville en 1956. Hormis la vallée inondée du Nyong qui occupe $9 \%$, l'espace restant (89\%), était réservé aux zones agricoles $(22 \%)$ parsemées à l'intérieur des forêts dégradées (qui occupaient $26 \%$ de l'espace), des forêts secondaires jeunes (23\%) et des forêts galeries (14\%) (figure4). 


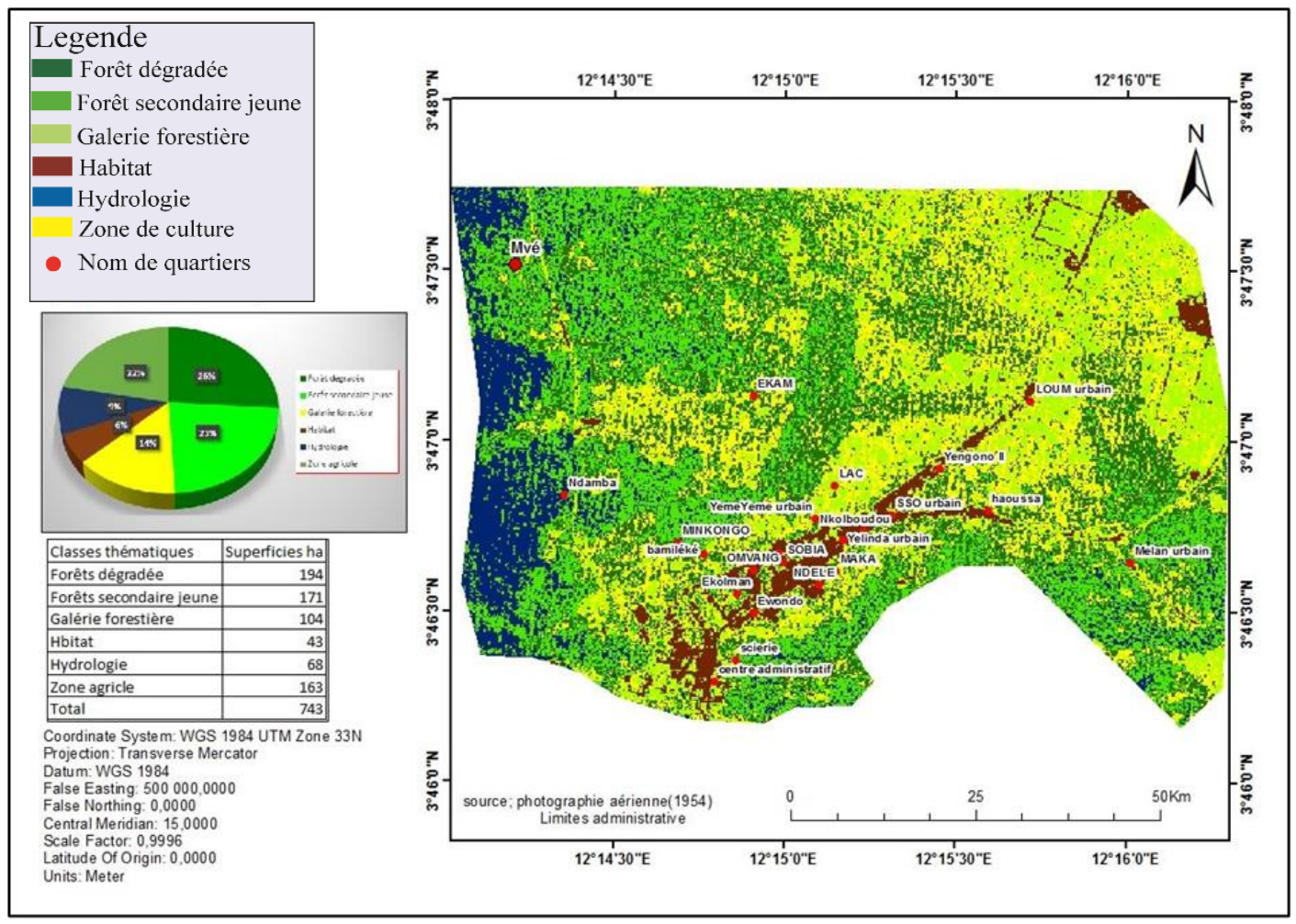

Figure 4 : Carte d'occupation des sols dans la ville d'Akonolinga en 1954

En 1999, l'espace urbain a connu une extension considérable. Cette mutation est susceptible d'être associée à la fois à son évolution administrative et à la construction de nombreuses infrastructures socioéconomiques. Il s'agit notamment de la création du Département du Nyong et Mfoumou avec pour Chef-lieu Akonolinga en 1964, de la construction du pont sur le Nyong inauguré en 1971 et reliant Akonolinga à Endom, du bitumage de la Route Yaoundé Ayos en 1992. Toutefois, entre 1954 et 1999, la superficie de cette ville, approchée par l'espace bâti, a été multipliée par un peu plus de 1,6 passant ainsi de 42 ha à 71 ha environ.

L'augmentation de la superficie s'est traduite sur le terrain par une extension urbaine à la fois au niveau des espaces disponibles dans le noyau originel qu'au niveau de la périphérie avec la colonisation de nouvelles terres vers le Nord-Ouest et le Nord-Est de la ville comme le montre la tache urbaine de 1999. C'est ainsi que sont nés les quartiers Lac, Akonolinga I, Bamiléké et le Centre administratif qui en plus des services administratifs, a vu arriver les constructions de bâtiments à usage d'habitation. Par ailleurs, les quartiers Ndamba, Mvé et Akonolinga se sont installés dans les nouveaux «fronts pionniers » de l'Est et de l'Ouest donnant ainsi naissance à ce que nous appelons la «nouvelle ville». 
En dépit de cet accroissement, l'analyse des taches urbaines (modélisation automatisée de l'emprise du tissu urbain) permet aussi d'observer que certaines zones densément peuplées en 1954 se sont dégradées au cours de cette période à l'instar de la partie Sud-Ouest de l'actuel quartier Sso. Cela peut être expliqué par la fermeture des usines de décorticage du café qui avaient attiré dans cette zone une importante population.

Du point de vue spatial, l'accroissement urbain ci-dessus décrit s'est accompagné d'une dynamique observable à travers les changements dans les modes d'occupation de l'espace. En effet, entre 1954 et 1999, on observe que la zone agricole qui entoure la ville est passée de 163 ha de superficie à 411 ha soit une augmentation en valeur relative de $252,15 \%$. Cet accroissement de la surface urbaine est le fruit d'une combinaison de deux facteurs que sont l'accroissement naturel et l'apport en migration. Cette augmentation de l'espace agricole s'est faite au détriment des autres formes de végétations qui jadis cohabitaient avec les cultures (Figure 5)

Figure 5 : Carte d'occupation des sols dans la ville d'Akonolinga en 1999

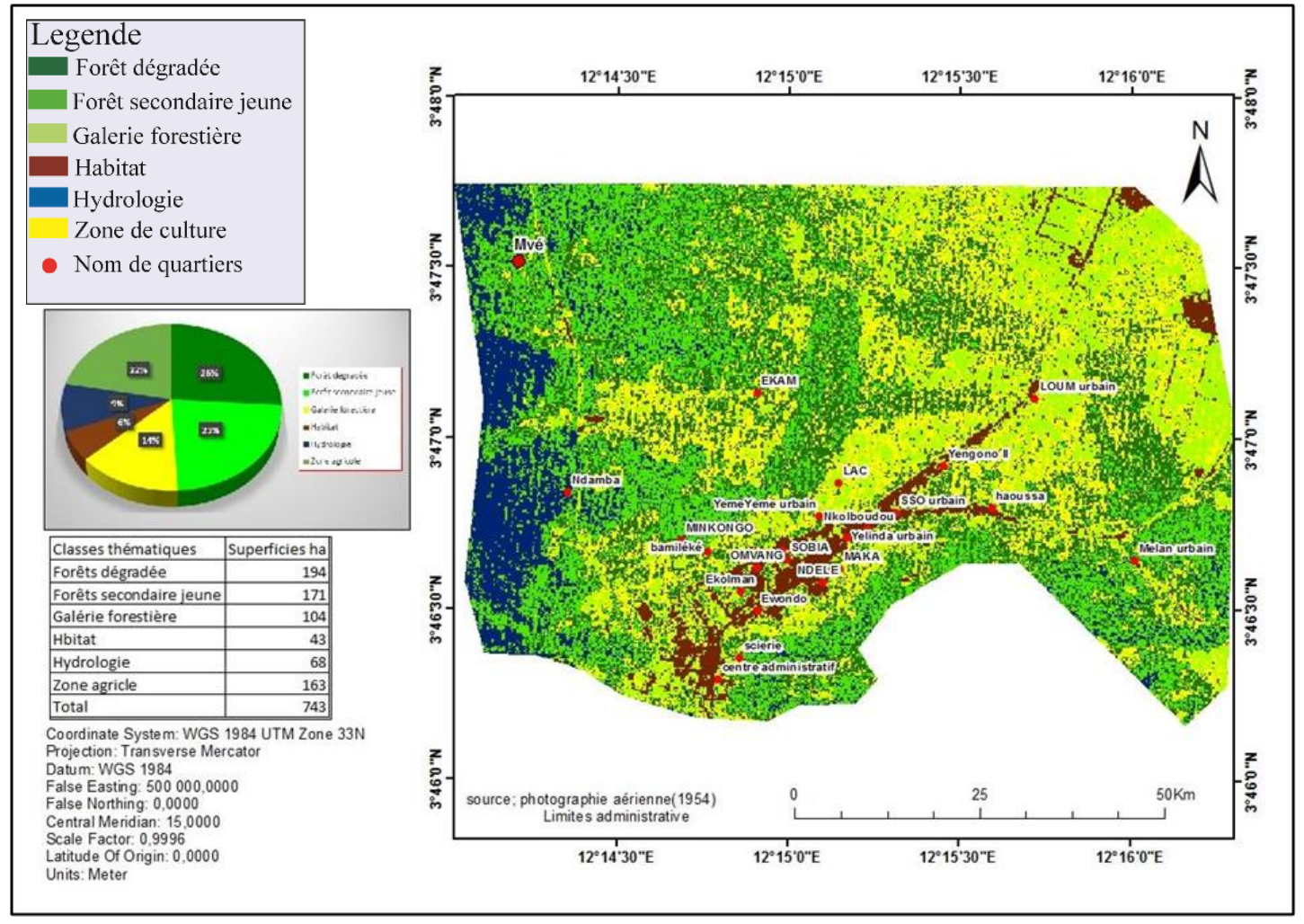

Les surfaces occupées par les galeries forestières, les zones inondées et les forêts dégradées se sont considérablement réduites. Par contre, les forêts secondaires jeunes ont vu leur superficie augmentée de 119 ha. Ces variations 
en matière d'occupation des sols et notamment la multiplication par 2,5 environ de l'espace occupé par la zone agricole et le quasi doublement de l'espace bâti traduisent une augmentation continue de la population d'Akonolinga au cours de la période 1954-1999. Cette augmentation serait liée à la poussée démographique des migrants et non migrants. A titre d'illustration, l'évolution démographique de la ville d'Akonolinga entre 1976 et 2017 montre une augmentation de 73,5\% de la population entre les deux périodes.

Entre 1999 et 2017, on observe une densification de la population dans la ville d'Akonolinga. Ce constat se justifie par un accroissement de l'espace urbain qui recule toujours un peu plus les limites de la ville. Au cours de cette période, l'espace bâti de la ville d'Akonolinga a vu sa superficie être multipliée par 4, 41 passant ainsi de 71 ha pour se situer à 313 ha en 2017 (Figure 6). Cette densification se caractérise par la fusion des trois grandes entités qui constituent la «ville nouvelle » en un bloc continu formant une sorte d'arc dirigée de l'Ouest vers le Nord-Nord-Est autour de la vielle ville. Par ailleurs on observe la création d'un nouveau « front pionnier » par-delà cet espace récemment urbanisé.

Au cours de la période 1999-2017, la croissance urbaine observée dans la ville d'Akonolinga va de pair avec une dynamique de l'espace qui se caractérise par le déclin de certaines entités naturelles. Ainsi, la surface couverte par les forêts secondaires jeunes a perdu 8 ha passant ainsi de 290 ha 282 ha. La forêt dégradée a quant à elle a été divisée par un peu plus de 8 pour se situer à 21 ha. De même, l'espace réservé à l'agriculture périurbaine a été divisé par deux et ne comptait plus que 250 ha. Enfin, la zone réservée aux galeries forestières a connu une légère augmentation ( $53 \mathrm{ha})$ tandis que la zone inondée se réduit considérablement et est en passe de disparaitre.

La dynamique de cet espace urbain entre 1999 et 2017 se caractérise essentiellement par une densification de l'habitat qui peut être corrélée à l'augmentation continue de sa population d'une part et à la migration d'autre part. En effet, l'analyse de l'évolution des caractéristiques des populations de cette ville selon le statut migratoire laisse entrevoir une relative prééminence des non-migrants sur les -migrants en 2017. En dépit de cette évolution, il se dégage une constante à savoir, les cours d'eau (Nyong et ses affluents) constituent de véritables obstacles à l'extension de la ville en direction du sud et du Sud-Ouest. A cette constante s'accompagne le dépeuplement du Sud-Est (figure 6). 
Figure 6 : Carte d'occupation des sols dans la ville d'Akonolinga en 2017
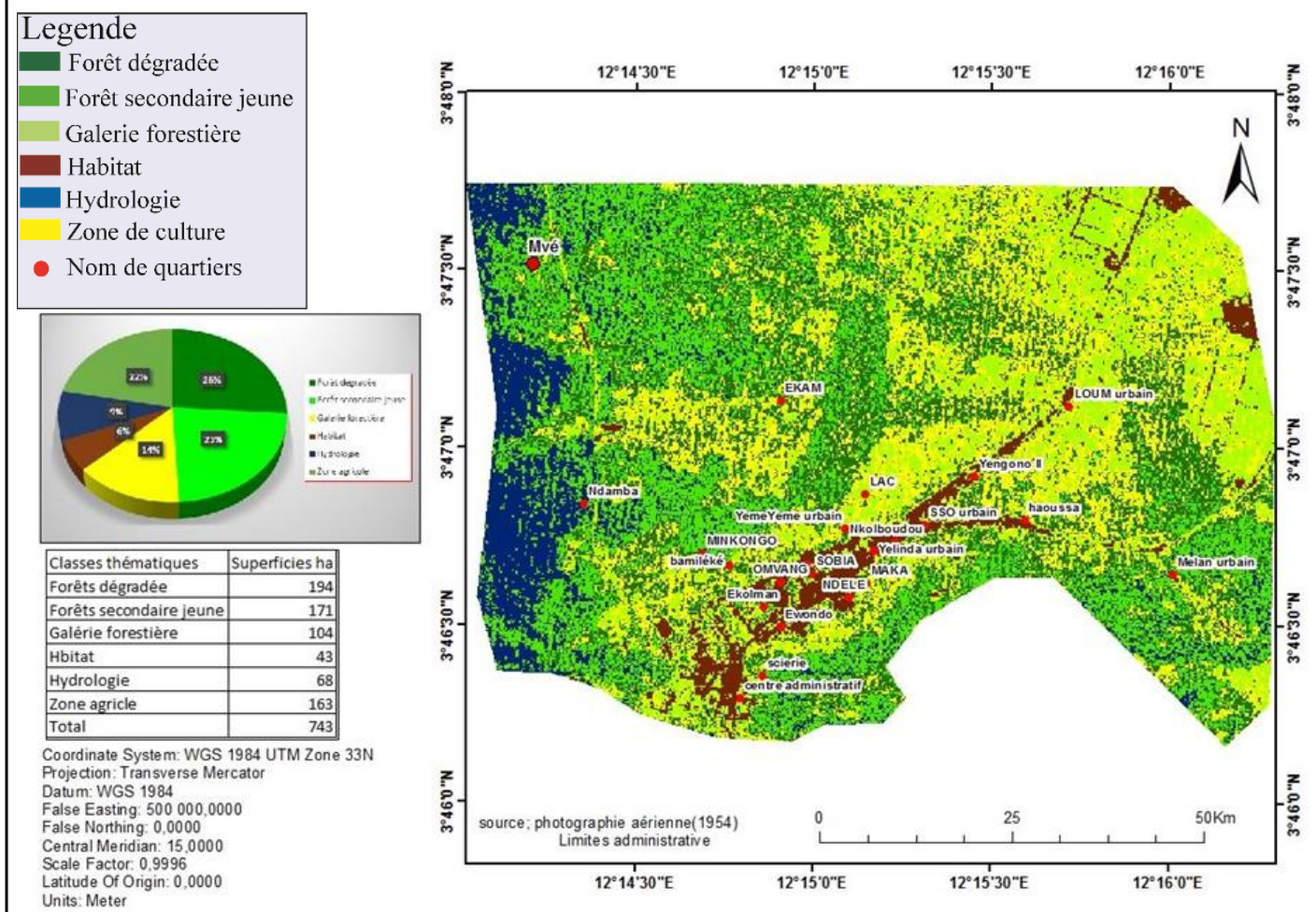

A l'issue de cette analyse, il se dégage le constat selon lequel l'expansion spatiale de la ville d'Akonolinga se fait dans le même sens que l'évolution des effectifs de la population en général et de ceux des migrants en particulier. La croissance démographique est responsable de cette dynamique de l'espace observée à Akonolinga. Les migrants participent aussi à structuration socio-économique des zones périphériques à cause de la disponibilité en ressources foncières dans ces espaces.

Toutefois, la quantification de cette contribution reste à déterminer de manière précise dans la mesure où d'autres facteurs notamment les changements des lieux de résidence intra-urbains des non-migrants contribuent également à comprendre cette dynamique spatiale. En outre, la dynamique de cet espace urbain est à l'origine de la dégradation du couvert végétal et de la déforestation. En effet, Rudel a montré en 2009 que l'augmentation de la population résultant d'un apport massif et exogène peut déclencher un processus de déforestation lié au changement de perception sur la forêt considérée comme une source de bois et de terres agricoles. Cette situation se prête à suffisance à la situation d'Akonolinga où l'essentiel de la population utilise le bois de chauffage comme principale source d'énergie pour la cuisson des aliments. 


\section{Contexte économique de la ville d'Akonolinga}

Avant de présenter la contribution de la migration à la pauvreté, faisons d'abord un aperçu général de la situation économique de la ville d'Akonolinga. Malgré des potentialités économiques importantes issues de son milieux physique (un climat favorable au développement des activités agricoles, des terres fertiles, de nombreux cours d'eau poissonneux, des forêts giboyeuses, les ressources minières...) Akonolinga se caractérise par une économie peu diversifiée et en déclin (Otsomotsi 2017). Jadis plaque tournante de l'économie du Département grâce à une activité économique basée sur la commercialisation du cacao et du café, l'exploitation et la transformation de son riche potentiel forestier par les entreprises forestières qui y avaient installé des scieries d'autre part. Aujourd'hui, Akonolinga ne dispose pas d'industrie, ni d'usine de transformation ou de pré-transformation des produits locaux. Son économie repose sur l'agriculture dont les principaux produits sont les tubercules (manioc, macabo, igname, ) la banane plantain, les cultures de rente (cacao, café, palmier à huile).

Le Plan Communal de Développent (2013) indique que les cultures de rente se pratiquent pour l'essentiel dans les périphéries urbaines et représente près de $70 \%$ de la production totale de la ville. La pêche artisanale, pratiquée essentiellement sur le Nyong, constitue le deuxième maillon de la production économique de cette ville et représente près de $20 \%$ de l'économie de la ville. Ces activités concernent l'ensemble de l'arrondissement. En marge de ces activités principales qui concentrent à elles seules l'essentiel de l'économique de la ville se développent quelques activités du secteur secondaire et tertiaire notamment les petites menuiseries qui transforment le bois débité en mobilier de maison, les activités de commerce de gros et de détail, l'artisanat, le tourisme, le transport intra et inter urbain et les activités liées à la fonction administrative de la ville (services départementaux des administrations publiques...).

Par ailleurs, dans l'espace urbanisé de cette petite ville se développe une activité économique portée par une kyrielle d'acteurs du secteur informel non agricole (les revendeuses des produits agricoles bruts, les pousseurs, les braiseuses de poissons, les call- boxeurs, les garagistes, les moto-taximen, les tenants de structures d'accueil et de restauration (auberges, hôtels, motels), les boutiquiers, etc). L'activité économique de cette ville fait face à de nombreuses difficultés parmi lesquelles l'insuffisance des infrastructures marchandes, le mauvais état de la voirie, l'insécurité, le faible accès à l'énergie et à l'eau. Les conséquences immédiates sont: la faiblesse des revenus des populations et des ménages, la précarité des emplois, le chômage qui touche environ $65 \%$ et une pauvreté persistante liée à la déstructuration du système de production économique, comme le relève Otsomotsi (2017, op.cit). 


\section{Activité économique des migrants à Akonolinga}

Deux variables retiennent notre attention dans le cadre de l'analyse du comportement économique des migrants à Akonolinga. Il s'agit du secteur d'activité et du statut dans l'emploi. S'agissant du secteur d'activité, les données contenues dans le tableau -ci-après montrent que la population de la ville d'Akonolinga exerce ses activités prioritairement dans le secteur tertiaire $(68 \%)$. Ce secteur est suivi par le secteur primaire qui occupe $32 \%$ des effectifs. Le secteur secondaire est quasi inexistant et ne représente que $2 \%$ des effectifs des personnes enquêtées (tableau 2).

Tableau 2 : Secteur d'activité selon le statut migratoire en 2017

\begin{tabular}{|c|c|c|c|c|c|c|}
\hline \multirow{3}{*}{ Secteur d'activité } & \multicolumn{6}{|c|}{ Statut migratoire } \\
\hline & \multicolumn{2}{|c|}{ Non-migrants } & \multicolumn{2}{|l|}{ Migrants } & \multicolumn{2}{|l|}{ Ensemble } \\
\hline & Effectifs & $\%$ & Effectifs & $\%$ & Effectifs & $\%$ \\
\hline Agriculture & 41 & $20 \%$ & 31 & $14 \%$ & 72 & $17 \%$ \\
\hline Elelvage & 9 & $4 \%$ & 16 & $7 \%$ & 25 & $6 \%$ \\
\hline Pêche et pisciculture & 10 & $5 \%$ & 6 & $3 \%$ & 16 & $4 \%$ \\
\hline Exploitation forestière & 2 & $1 \%$ & 5 & $2 \%$ & 7 & $2 \%$ \\
\hline Extraction & 6 & $3 \%$ & 4 & $2 \%$ & 10 & $2 \%$ \\
\hline Industries alimentaire & 4 & $2 \%$ & 5 & $2 \%$ & 9 & $2 \%$ \\
\hline Electricité eau et gaz & 3 & $1 \%$ & 4 & $2 \%$ & 7 & $2 \%$ \\
\hline Administration & 30 & $15 \%$ & 46 & $20 \%$ & 76 & $18 \%$ \\
\hline Construction & 15 & $7 \%$ & 13 & $6 \%$ & 28 & $7 \%$ \\
\hline Commerce & 59 & $29 \%$ & 57 & $25 \%$ & 116 & $27 \%$ \\
\hline Transport & 12 & $6 \%$ & 23 & $10 \%$ & 35 & $8 \%$ \\
\hline Coiffeuse/Coiffure & 3 & $1 \%$ & 11 & $5 \%$ & 14 & $3 \%$ \\
\hline Ménagère & 10 & $5 \%$ & 4 & $2 \%$ & 14 & $3 \%$ \\
\hline Total & 204 & $100 \%$ & 225 & $100 \%$ & 429 & $100 \%$ \\
\hline
\end{tabular}

Source : Nos analyses de données de l'enquête réalisée en 2017

En ce qui concerne spécifiquement l'activité économique des migrants, les résultats du tableau 2 montrent que la tendance observée dans l'ensemble de la population reste la même. En effet, les migrants exercent à $70 \%$ dans le secteur des services contre $65 \%$ pour les non-migrants. Les principales activités concernées sont le commerce (25\%), les services associés à la fonction administrative (20\%), le transport (10\%), la construction des édifices $(6 \%)$, la coiffure $(5 \%)$. Ces activités correspondent dans l'ensemble à celles du secteur informel non agricole et correspondent dans leur ensemble au contexte économique de l'espace urbanisé d'Akonolinga décrit plus haut. Les migrants sont plus nombreux dans le secteur des services du fait que pendant de très longues années, les populations autochtones se sont beaucoup focalisées sur l'agriculture. Le dynamisme socio-économique des migrants se manifeste aussi dans leur statut dans l'emploi. Il (tableau 3). 
Tableau 3 : Statut dans l'emploi selon le statut migratoire en 2017

\begin{tabular}{|l|l|l|l|l|l|l|}
\hline \multirow{2}{*}{$\begin{array}{l}\text { Statut dans } \\
\text { l'emploi }\end{array}$} & \multicolumn{4}{l}{ Statut migratoire } & \multicolumn{2}{l|}{ Ensemble } \\
\cline { 2 - 7 } & Non-Migrants & Migrants & \multicolumn{2}{l|}{} \\
\cline { 2 - 7 } & Effectif & $\mathbf{\%}$ & Effectif & $\%$ & Effectif & $\%$ \\
\hline $\begin{array}{l}\text { Employeur/ } \\
\text { Indépendant }\end{array}$ & 108 & $53 \%$ & 121 & $52 \%$ & 229 & $53 \%$ \\
\hline Salarié & 63 & $31 \%$ & 78 & $34 \%$ & 141 & $32 \%$ \\
\hline Aide familial & 25 & $12 \%$ & 28 & $12 \%$ & 53 & $12 \%$ \\
\hline Apprenti & 4 & $2 \%$ & 5 & $2 \%$ & 9 & $2 \%$ \\
\hline Retraité & 3 & $1 \%$ & 0 & $0 \%$ & 3 & $1 \%$ \\
\hline Total & 203 & $100 \%$ & 232 & $100 \%$ & 435 & $100 \%$ \\
\hline
\end{tabular}

Source : Nos analyses des données de l'enquête réalisée en 2017

Le tableau 3 indique le statut dans l'emploi selon le statut migratoire en 2017. L'analyse des statistiques indiquent que les migrants manifestent un dynamisme socio-économique par rapport aux non-migrants. A titre d'illustration, les observations suivantes indiquent que :

- Au niveau du statut dans l'emploi en tant qu'employeur/ indépendant, les statistiques indiquent $52,83 \%$ de migrants contre $47,17 \%$ de nonmigrants ;

- Quant au statut salarié, le constat de terrain indique 55,31\% de migrants contre $44,69 \%$ pour les non-migrants ;

- Le volet aide familial fait intervenir $52,83 \%$ des migrants contre $47,17 \%$ pour les non-migrants ;

- Les migrants s'illustrent aussi dans les activités d'apprentissage des métiers $(55,55 \%)$ alors que les non-migrants s'activent à 44,45\% ;

- L'on note plus de retraité chez les non-migrants (100\%) que chez les migrants.

\section{Discussion}

$\mathrm{Au}$ regard de ces résultats, nous pouvons dire qu'en dépit de l'inexistence des données pour les autres années d'observation, les migrants contribuent à la dynamique de l'espace urbain à Akonolinga et à l'accroissement de la pauvreté. La contribution de la migration à la dynamique spatiale a été observée dans le cadre de nombreuses autres études et constitue d'ailleurs un phénomène ancien. Watang (2014) imputait déjà la croissance spatiale des petites villes agricoles (Maga, Pitoa, Lagdo, Ngong, Tcheboa, Touboro) à l'immigration. En Dabs cet ordre d'idéé Roupsad (1987) souligne aussi que :

«Les vallées du Logone (Extrême-Nord) et de la Bénoué (région du Nord) ont bénéficié des aménagements agricoles depuis les années 1970. La croissance de la production agricole qui en résulta a favorisé le 
développement des marchés de collecte et autres activités commerciales. Plus de 200000 migrants internes se sont installés dans ces pôles agricoles (Roupsard, 1987)».

Il en est de même pour la contribution de la migration à l'accroissement de la pauvreté. En effet, selon les résultats obtenus, les migrants ont une influence sur la pauvreté à Akonolinga. En effet, ils y contribuent de manière significative. Ces résultats sont cohérents avec ceux obtenus par d'autres chercheurs. A titre illustratif, selon une étude de Christiaensen (2012) rapportée dans le monde.fr par Jacquet et relative à l'impact de l'exode rural sur la pauvreté : «La migration vers les villes s'accompagne d'une accélération de la croissance. Mais elle coexiste avec un creusement des inégalités et même souvent un accroissement de la pauvreté».

A la lumière de ces résultats, nous pouvons dire que l'hypothèse selon laquelle « la dynamique spatiale observée à Akonolinga est le fait des migrants » a été confirmée. Toutefois, la dynamique de l'espace urbain devra encore être précisée dans le cadre d'études quantitatives plus spécifiques visant à isoler les contributions respectives des migrants et des non migrants à la dynamique spatiale. Ceci est d'autant plus vrai que certaines personnes interviewées à Akonolinga estiment que les mutations spatiales observées sont également le fait des non-migrants qui cherchent à satisfaire les besoins en logement des migrants de plus en plus nombreux en construisant des maisons dans les zones anciennement réservées à l'agriculture périurbaine. C'est notamment le cas de Sa Majesté Béti Joseph, Chef du quartier Minkongo-Ekoudou pour qui : "Ce sont les immigrants qui poussent les autochtones à construire pour pouvoir les louer leurs maisons ». Donc les migrants à créer les richesses dans la ville d'Akonominga.

Par ailleurs, les données des recensements généraux de la population du Cameroun utilisées présentaient des limites liées pour l'essentiel à la variation des informations collectées d'une année à l'autre et ne permettaient pas de faire des analyses sur la période de l'étude. Ces données sont aussi obsolètes et demeurent questionnables. En outre, la mesure de la pauvreté dans la présente étude est également insuffisante. En effet, si à base des éléments du logement, de l'assainissement et des méthodes statistiques nous sommes parvenus à construire objectivement un indicateur de pauvreté au niveau de la ville d'Akonolinga, il n'en demeure pas moins vrai que dans la perception et la réalité, la pauvreté associe à ces éléments objectifs une bonne dose d'émotions, de sentiments et d'histoire de vie qui dépassent le cadre " réducteur de la classe statistique ». Surtout lorsqu'on sait que car de nombreuses méthodes d'approximation de la pauvreté existent et que cellesci prennent en compte de nombreuses variables autres que celles que nous avons utilisées. 


\section{Conclusion}

De l'analyse des interactions mobilités, dynamique de l'espace et pauvreté à Akonolinga, il ressort que l'immigration contribue à la dynamique de l'espace urbanisé de cette petite ville ainsi qu'à l'accroissement de la pauvreté. Les déplacements des migrants à l'intérieur de la ville montrent que les quartiers périphériques ont bénéficié de leur apport. En effet, les migrants qui se sont installées dans les quartiers centraux et péricentraux à leur arrivée résident en 2017 dans les quartiers périphériques. Ces migrants du fait de leur dynamisme contribuent à la création des richesses à Akonolinga d'une part et d'autre part ils sont les acteurs de l'extension des périphéries de la ville. Les villes moyennes du Cameroun comme celle d'Akonolinga ne disposent pas assez d'infrastructures socio-économiques pour accueillir un nombre important de nouveaux migrants. Plusieurs stratégies sont à implémentées pour venir à bout de la migration interne au Cameroun : (i) limiter les déplacements des populations des zones rurales vers les zones urbaines et ; (ii) stabiliser les populations dans les centres urbains secondaires en vue de réduire l'engorgement des grandes villes. Ces stratégies pourront déboucher sur la définition de politiques et programmes plus adaptés et à même de promouvoir un développement socioéconomique plus équilibré entre les régions du pays et permettre à toutes les populations où qu'elles soient, d'avoir accès, dans les mêmes conditions, aux biens et services essentiels et indispensables à une vie décente. L'étude des phénomènes migratoires et ses incidences spatiales et socio-économiques doit permettre d'opérationnaliser le concept de mobilité spatiale dans la mise en œuvre des divers plans d'aménagement du territoire.

\section{References:}

1. Abêt Mongbet (2019). Mobilités, dynamiques frontalières et intégration sous-régionale en zone CEMAC : le cas des commerçants de Kyé-Ossi , Thèse de Doctorat, Université de Poitier , 418 p

2. Bakewell Olivier et Gunvor Jónsson (2011). Migration, mobilité et villes africaines . Rapport de synthèse sur le programme de recherche Perspectives africaines sur la mobilité humaine

3. BUCREP., (2010). Rapport de présentation des résultats définitifs du Troisième Recensement General de La Population et de l'habitat, Yaoundé, BUCREP, 63p.

4. BUCREP., (2010). $3^{\text {ème }}$ RGPH, vol 2, tome 9, Mouvement migratoire, Yaoundé, BUCREP, 307 p.

5. BUCREP., (2010). $3^{\text {ème }}$ RGPH, volume 2, tome 14, Mesure et cartographie de la pauvreté à partir des conditions de vie, Yaoundé, BUCREP, $241 \mathrm{p}$. 
6. Christiaensen L., Olaberria E., et Rigolin J., (2012). Natural disasters and growth: Going beyond the averages, in World Development, Vol 40, Issue 7, July 2012, pp 1317-1336.

7. Conférence des Nations Unies sur le Commerce et le Développement (2018). Le développement économique en Afrique : les migrations au service de la transformation structurelle, $11 \mathrm{p}$

8. Dominique T., et Schoumaker B. (2009). Démographie et région du monde : Etat et tendance, La démographie de l'Afrique au sud du Sahara des années 1950 aux années $2000 »$ p. 357-381.

9. INS., (2005). Enquête sur l'Emploi et Secteur Informel au Cameroun, rapport principal, Yaoundé, INS, $200 \mathrm{p}$.

10. INS., (2011). Enquête démographique et de santé et à indicateurs multiples, Rapport principal Yaoundé, INS, $546 \mathrm{p}$.

11. INS., (2011). Enquête démographique et de santé et à indicateurs multiples, Yaoundé, INS, $546 \mathrm{p}$.

12. INS., (2015). Tendance, profil et déterminants de la pauvreté au Cameroun entre 2001 et 2014, rapport principal, INS, 72 p.

13. Jacquet P. (2012), L'exode rural, un impact discuté sur la pauvreté, in http://www.lemonde.fr/economie/article/2012/06/25/l-exode-ruralun-impact-discute-sur-la-

pauvrete_1724090_3234.html\#1QV20XduVvOzgI0j.99, consulté le 16 janvier 2018 à $18 \mathrm{~h} 00$

14. Kobiané J-F., (1998). Essai de construction d'un profil de pauvreté des ménages à Ouagadougou à partir des caractéristiques de l'habitat, in : Francis Gendreau (éd.), Crises, pauvreté et changements démographiques dans les pays du Sud, AUPELF-UREF, Éditions ESTEM, Paris, pp 117-131.

15. OIM., (2009). Migration au Cameroun : profil national, OIM, $128 \mathrm{p}$.

16. Otsomotsi M A., (2010). Les déterminants sociodémographiques de la qualité du logement dans les métropoles camerounaises : cas de Douala et de Yaoundé, mémoire de master professionnel en démographie, Institut de Formation et de Recherche Démographiques, Université de Yaoundé 2, $147 \mathrm{p}$.

17. Paugam S., (2005). Les formes élémentaires de la pauvreté, Paris, Presses Universitaires de France, coll. «Le lien social », 312 p.

18. Plan Communal de Développement d'Akonolinga. (2013). Commune d'Akonolinga, Tome 1, $218 \mathrm{p}$.

19. Richard Danziger (2019). les défis de la migration en Afrique de l'Ouest et du Centre, in www.google.fr

20. Roupsard Marcel (1987). Nord Cameroun : entre ouverture et développement, thèse de Doctorat en géographie, université, Université de Paris -Sorbonne 
21. Rudel T. Defries R., Asner G P., \& Laurance W F., (2009). Changing drivers of deforestation and new opportunities for conservation. Conserv. Biol., 23(6), 1396-1405.

22. Tolno D., et Soultan I A., (2008). "Qualité et confort des logements dans la ville de Djibouti », in Belbeoc O, La population de Djibouti, L'harmattan, Paris, pp 245-266.

23. Sandron F. (2007) . Impact des migrations sur l'environnement, in Grain de sel $n^{\circ} 40$ septembre- novembre 2007

24. Teye Joseph et Mariama Awumbila (2020). Facteurs de migration et d'urbanisation en Afrique, in www.google.fr

25. Veron J. (2008). Enjeux économiques, sociaux et environnementaux de l'urbanisation du monde, in Mondes en développement $\mathrm{n}^{\circ} 142$ 2008/2, pp. 39-52.DOI 10.3917/med.142.0039

26. Watang Z F., (2014). Immigration, croissance démographique et dynamique urbaine au Nord Cameroun, in African Population Studies, Vol. 28, No. 3, pp 1233-12 